\title{
Estimating the burden of acute gastrointestinal illness due to Giardia, Cryptosporidium, Campylobacter, E. coli 0157 and norovirus associated with private wells and small water systems in Canada
}

\author{
H. M. MURPHY ${ }^{1}$, M. K. THOMAS ${ }^{1}$, P. J. SCHMIDT ${ }^{2}$, D. T. MEDEIROS ${ }^{3}$, \\ S. MCFADYEN ${ }^{3}$ AND K. D. M. PINTAR ${ }^{1 *}$ \\ ${ }^{1}$ Centre for Food-borne, Environmental and Zoonotic Infectious Diseases, Public Health Agency of Canada, \\ Guelph, ON, Canada \\ ${ }^{2}$ Department of Civil and Environmental Engineering, University of Waterloo, Waterloo, ON, Canada \\ ${ }^{3}$ Water and Air Quality Bureau, Healthy Environments and Consumer Safety Branch, Health Canada, Ottawa, \\ ON, Canada
}

Received 26 March 2015; Final revision 13 July 2015; Accepted 21 August 2015; first published online 13 November 2015

\section{SUMMARY}

Waterborne illness related to the consumption of contaminated or inadequately treated water is a global public health concern. Although the magnitude of drinking water-related illnesses in developed countries is lower than that observed in developing regions of the world, drinking water is still responsible for a proportion of all cases of acute gastrointestinal illness (AGI) in Canada. The estimated burden of endemic AGI in Canada is 20.5 million cases annually - this estimate accounts for under-reporting and under-diagnosis. About 4 million of these cases are domestically acquired and foodborne, yet the proportion of waterborne cases is unknown. There is evidence that individuals served by private systems and small community systems may be more at risk of waterborne illness than those served by municipal drinking water systems in Canada. However, little is known regarding the contribution of these systems to the overall drinking water-related AGI burden in Canada. Private water supplies serve an estimated $12 \%$ of the Canadian population, or $\sim 4.1$ million people. An estimated 1.4 million (4.1\%) people in Canada are served by small groundwater $(2 \cdot 6 \%)$ and surface water $(1 \cdot 5 \%)$ supplies. The objective of this research is to estimate the number of AGI cases attributable to water consumption from these supplies in Canada using a quantitative microbial risk assessment (QMRA) approach. This provides a framework for others to develop burden of waterborne illness estimates for small water supplies. A multi-pathogen QMRA of Giardia, Cryptosporidium, Campylobacter, E. coli $\mathrm{O} 157$ and norovirus, chosen as index waterborne pathogens, for various source water and treatment combinations was performed. It is estimated that 103230 AGI cases per year are due to the presence of these five pathogens in drinking water from private and small community water systems in Canada. In addition to providing a mechanism to assess the potential burden of AGI attributed to small systems and private well water in Canada, this research supports the use of QMRA as an effective source attribution tool when there is a lack of randomized controlled trial data to evaluate the public health risk of an exposure source. QMRA is also a powerful tool for identifying existing knowledge gaps on the national scale to inform future surveillance and research efforts.

Key words: Water (quality), waterborne illness, burden, small drinking water systems, private wells.

\footnotetext{
* Author for correspondence: Dr K. D. M. Pintar, Centre for Food-borne, Environmental and Zoonotic Infectious Diseases, Public Health Agency of Canada, 130 Colonnade Rd, Ottawa, ON, K1A 0K9. (Email: Katarina.Pintar@phac-aspc.gc.ca)

This is an Open Access article, distributed under the terms of the Creative Commons Attribution licence (http://creativecommons.org/licenses/by/4.0/), which permits unrestricted re-use, distribution, and reproduction in any medium, provided the original work is properly cited.
} 


\section{INTRODUCTION}

The magnitude and sources of waterborne (enteric) illness in Canada are not well-defined. Enteric illness is largely under-reported, and existing national and provincial surveillance systems for enteric illness do not distinguish between infections caused by food, animal contact, person-to-person, environmental, or drinking water transmission. The Public Health Agency of Canada estimates that there are roughly 20.5 million AGI cases each year ( $0 \cdot 6$ cases/person per year) [1]. Of the overall burden, we estimate that 4 million cases are foodborne (and acquired domestically) [1], while the remaining cases are attributed to water, animal contact, and person-to-person transmission.

Private water supplies (households) serve an estimated $12 \%$ of the Canadian population, or $\sim 4.1$ million people [2]. Eleven percent of Canadians are supplied by a private (unregulated) groundwater source, and $1 \%$ use a private surface water source (e.g. a spring, lake, river or dugout) [2]. The responsibility of managing and maintaining the quality of these water supplies falls to their owners [3]. Health Canada recommends that households on private wells have their water tested by a laboratory 2-3 times per year [4]; however, only $27 \%$ of households on private water supplies had their water tested in 2011 [2].

An estimated 1.7 million (4.9\%) Canadians are served by small community groundwater $(3 \cdot 1 \%)$ and surface water $(1.8 \%)$ supplies [2, 5]. For this study, a small supply is defined as a system serving $<1000$ people. Private and small community water systems may be more at risk for human illness than municipally operated systems in Canada [6-9]. In a review of Canadian waterborne outbreaks between 1974 and 2001, two-thirds of the outbreaks occurred at either private or semi-private systems [10]. Campylobacter spp., Cryptosporidium spp., Giardia spp., and E. coli O157 were responsible for the majority $(\sim 58 \%)$ of these outbreaks, which is consistent with European trends [11]. In addition, Schuster et al. [10] found that Norwalk-like viruses and rotavirus were responsible for $9 \cdot 3 \%$ (14/150) of Canadian outbreaks where causative organisms were identified. This is consistent with findings in the United States [12], where 6\% of waterborne outbreaks were due to norovirus.

There is some evidence to suggest that private well and small water system users may be at increased risk of AGI; however, the magnitude of this risk had not been quantified in Canada. The objective of the work presented herein is to estimate the number of AGI cases associated with Giardia, Cryptosporidium, Campylobacter, E. coli $\mathrm{O} 157$ and norovirus associated with the consumption of water from private and small community systems in Canada using a quantitative microbial risk assessment (QMRA) approach. This study is part of a comprehensive approach to quantify and attribute AGI in Canada to various sources to inform policy, research and surveillance efforts [13].

\section{MATERIALS AND METHODS}

\section{Model framework}

A multi-pathogen QMRA was developed using Giardia, Cryptosporidium, Campylobacter, E. coli $\mathrm{O} 157$ and norovirus as these are the pathogens most commonly associated with enteric drinking water outbreaks in Canada. Five stochastic models were developed to estimate the number of AGI cases caused by each pathogen, separated into three types of drinking water supplies: private groundwater wells, small groundwater supplies (serving $<1000$ individuals), and small surface water supplies (serving $<1000$ individuals). The QMRA methodology and subsequent equations applied were adapted from Haas et al. [14] and Howard et al. [15] (Fig. 1). The daily probability of infection in the present study represents the probability of being infected following exposure to a water supply that is contaminated with the pathogen of interest. The annual probability of infection represents the probability of infection over a 1-year period accounting for the possibility of exposure to a water supply that is positive for pathogens (prevalence rate). In this analysis, the conversion from probability of illness to cases of illness assumed a maximum of one illness per year.

QMRA was used because no randomized control trials have been performed on small community supplies or private wells [16]. To capture the uncertainty and variability associated with the estimates, inputs were described using probability distributions. The minimum, maximum, and most likely values for the variables were developed (using PERT and Uniform distributions, or raw data fit to parametric distributions such as Lognormal or Weibull) [17]. The final estimates [reported as mean with $90 \%$ probability interval (PI) around the mean] were generated using Monte Carlo simulation[10000 iterations using @Risk (Palisade Corp., USA)]. Sensitivity analyses were performed by examining the Spearman correlation coefficients $\left(r_{s}\right)$. 


\section{where:}

$$
\begin{aligned}
& \text { Daily exposure }\left(E_{\mathrm{d}}\right) \\
& E_{\mathrm{d}}=C * 1 / R * I * 10^{-\mathrm{LR}} * V
\end{aligned}
$$

$C=$ concentration of pathogen (organisms/litre)

$R=$ mean analytical recovery

$I=$ fraction of pathogens that are infectious (used for Cryptosporidium only)

$\mathrm{LR}=$ log-reduction by treatment applied in small water systems

$V=$ daily volume of water consumed by population at risk (I/day), separating children, adults and elderly

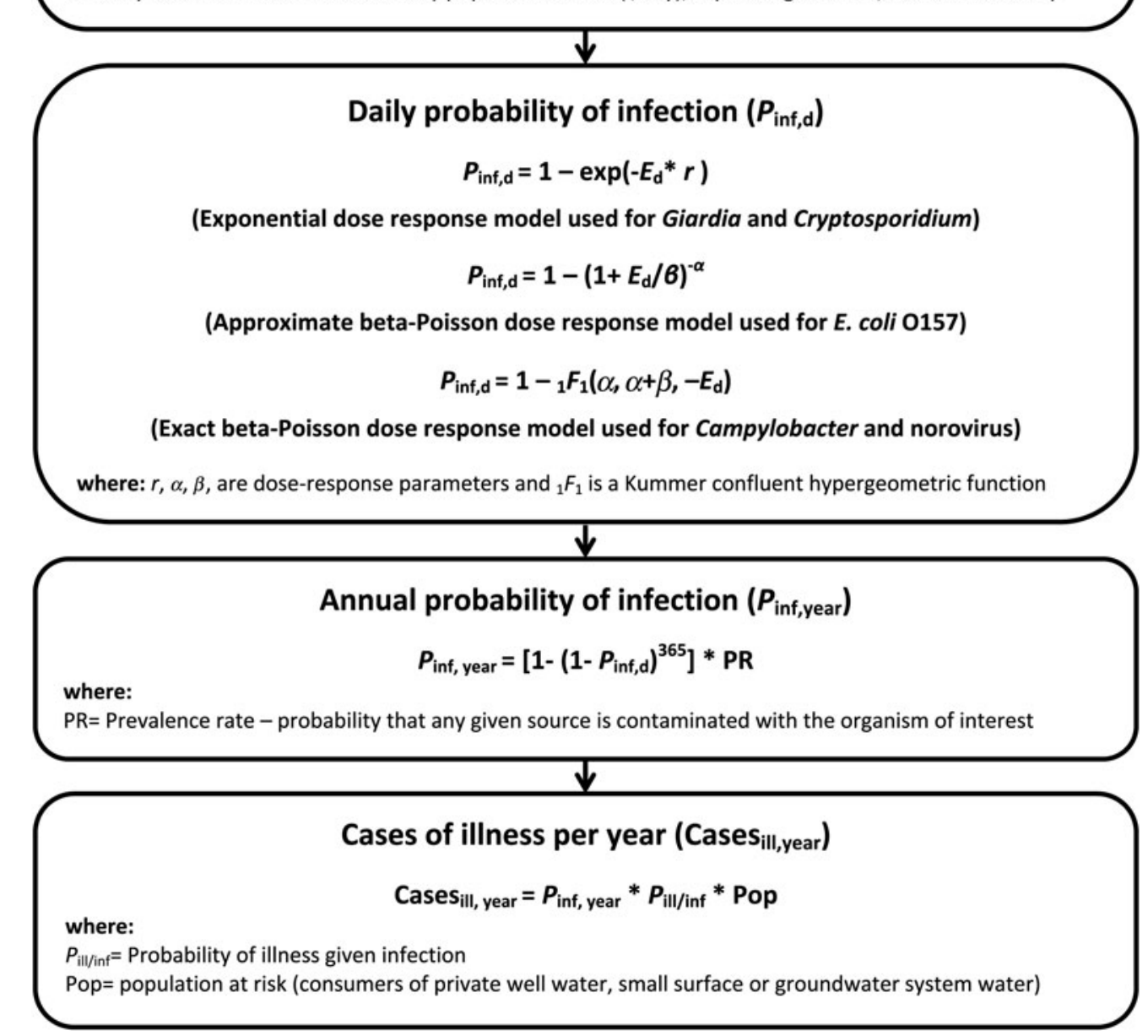

Fig. 1. Schematic diagram of QMRA model used to estimate the disease burden of Giardia, Cryptosporidium, Campylobacter, E. coli $\mathrm{O} 157$ and norovirus from the consumption of water from private wells and small water systems serving <1000 people (adapted from Haas et al. [14]; Howard et al. [15]).

\section{Model inputs}

\section{Population at risk}

Using the 2011 Canadian population (34342 800) [18], and data on the proportion of Canadians that consumed water from private and small system groundwater and surface water supplies [2], the approximate population on each of these water supplies was estimated: 1068830 individuals on small municipal groundwater systems, 614128 individuals on small surface water systems, 4138080 individuals on private wells, and 22246976 individuals on large municipal systems. The proportion of Canadians that report exclusively consuming bottled water and the proportion of households that reportedly treat their water at the intake to their home and state that this is to treat for bacteria [2] were excluded (Table 1). The proportion of children ( $\leqslant 10$ years), adults (11-64 years) and elderly $\geqslant 65$ years) were estimated [18] to generate age-specific incidence rates (Table 2). 
Table 1. Estimation of the population at risk that consumes water from private wells and small system supplies in Canada

\begin{tabular}{|c|c|c|c|c|}
\hline Population category & Private wells & Small GW systems & Small SW systems & Total \\
\hline A. Total population & 4138080 & 1068830 & 614128 & 5821038 \\
\hline $\begin{array}{l}\text { B. Proportion of people that drink } \\
\text { bottled water exclusively }\left(27 \% \%^{*} \text {, }\right. \\
22 \% \dagger \text { of A) }\end{array}$ & 1117282 & 235143 & 135108 & 1487533 \\
\hline $\begin{array}{l}\text { C. Proportion of tap water households } \\
\text { that treat their water at intake to } \\
\text { home }\left[34^{*}, 5 \% \dagger \text { of }(\mathrm{A}-\mathrm{B})\right]\end{array}$ & 1027071 & 41684 & 23951 & 1092706 \\
\hline $\begin{array}{l}\text { D. Estimated proportion of } \\
\text { households that treat for bacteria } \\
(31 \% *, 32 \% \dagger \text { of C) }\end{array}$ & 318392 & 13339 & 7664 & 339395 \\
\hline $\begin{array}{l}\text { E. Distribution of population at risk: } \\
\text { Uniform }[(\mathrm{A}-\mathrm{B}-\mathrm{D}) ;(\mathrm{A}-\mathrm{B})]\end{array}$ & (2 702 406-3 020798$)$ & (820 348-833 687) & (471 356-479 020) & \\
\hline
\end{tabular}

GW, Groundwater; SW, surface water.

* Applies to private wells.

$\dagger$ Applies to small GW and small SW systems.

Table 2. Population served by private wells and small system water supplies in Canada, and water consumption distribution inputs by age group (children, adults, elderly)

\begin{tabular}{|c|c|c|c|c|}
\hline \multirow[b]{2}{*}{ Population category* } & \multicolumn{3}{|c|}{ Estimated population ranges (minimum-maximum) } & \multirow{2}{*}{$\begin{array}{l}\text { Water consumption } \\
\text { distributions (1/day) } \\
\text { lognormal, mean (s.D.) }\end{array}$} \\
\hline & Private wells & Small GW systems & Small SW systems & \\
\hline Children $(\leqslant 10$ years) & $462111-516556$ & $140280-142560$ & $80602-81912$ & $1 \cdot 207(0.632)$ \\
\hline Adults (11-64 years) & $1756564-1963519$ & $533226-541897$ & $306381-311363$ & $1 \cdot 500(0 \cdot 924)$ \\
\hline Elderly ( $\geqslant 65$ years) & $483731-540723$ & $146842-149230$ & $84373-85745$ & $1 \cdot 260(0 \cdot 650)$ \\
\hline
\end{tabular}

GW, Groundwater; SW, surface water.

* Age categories in the 2011 census (Statistics Canada [5]) were $\leqslant 10$ years for children (17·1\%); 15-64 years for adults (65\%); $\geqslant 65$ years for the elderly $(17 \cdot 9 \%)$. No adjustments were made to reconcile these census categories with the water consumption categories.

\section{Water consumption}

Water consumption data collected from a Canadian community survey were used to develop consumption inputs for three age groups: $\leqslant 10$ years $(n=132)$, $11-64$ years $(n=1636)$ and $\geqslant 65$ years $(n=331)$ (raw data from [19]), and fit to a lognormal distribution [20].

\section{Canadian groundwater and surface water pathogen} occurrence and concentration data

All inputs for both pathogen prevalence rates (PR) and concentration $(C)$ in groundwater [21] and surface water were derived from the published literature (Tables 3, 4; Supplementary Tables S1, S2). Data were obtained from those studies that examined pathogen occurrence under 'non-outbreak' conditions. Canadian pathogen data were included where available. In the absence of Canadian data, US studies were used. Where Canadian or US data were unavailable, international studies were used. It was assumed that reported non-detects or zero values from the literature were zero.

For small groundwater and surface water systems, the level of treatment applied (log reduction) was considered based on the treatment systems currently in place in Canada (Fig. 1). Using data from the Survey of Drinking Water Plants [5], 19 treatment types were developed (Supplementary Table S3: Treatment types). These treatment types were further combined into the following five categories.

(1) No treatment.

(2) Membrane filtration (micro- or ultra-filtration) with chemical disinfection (with/without other treatment, but no UV disinfection).

(3) Media filtration (with/without other treatment, but no UV or membrane filtration). 
Table 3. Groundwater pathogen inputs selected for use in the private well and small groundwater system QMRA models for Giardia, Cryptosporidium, Campylobacter, E. coli O157 and norovirus

\begin{tabular}{|c|c|c|c|}
\hline Model parameter & Distribution & Rationale & Reference \\
\hline \multicolumn{4}{|l|}{ Giardia } \\
\hline Prevalence rate & $\begin{array}{l}\text { Point estimate } \\
(1 \cdot 34 \%)\end{array}$ & $\begin{array}{l}1 \cdot 34 \% \text { of wells were positive with Giardia in US } \\
\text { study; nearly all other US/Canada studies reported } \\
\text { no detection of Giardia in well water }\end{array}$ & Hancock et al. [48] \\
\hline $\begin{array}{l}\text { Concentration } \\
\text { (cysts/l) }\end{array}$ & $\begin{array}{l}\text { Lognormal* } \\
(0 \cdot 01,0 \cdot 025)\end{array}$ & $\begin{array}{l}\text { Data were obtained from one paper, which collected } \\
253 \text { samples from } 149 \text { wells across the United States } \\
\text { in different geographical locations (only study of this } \\
\text { magnitude in North America). Reported median } \\
\text { substituted for arithmetic mean }\end{array}$ & Hancock et al. [48] \\
\hline Recovery & $\begin{array}{l}\text { Point estimate } \\
(47 \%)\end{array}$ & Middle of range reported by Hancock et al. [48] & Hancock et al. [48] \\
\hline \multicolumn{4}{|l|}{ Cryptosporidium } \\
\hline Prevalence rate & $\begin{array}{l}\text { PERT: } \\
(0 \%, 4 \cdot 7 \%, 6 \cdot 2 \%)\end{array}$ & $\begin{array}{l}0 \% \text { is the lowest occurrence from Isaac-Renton et al. } \\
{[62] ; 4 \cdot 7 \% \text { is the prevalence from Hancock et al. }[48]} \\
\text { because it is the largest published study; } 6 \cdot 2 \% \text { is } \\
\text { based on pooled US/Canada positive-well results (i.e. } \\
12 / 193 \text { ) }\end{array}$ & $\begin{array}{l}\text { Hancock et al. }[48] \text {; } \\
\text { Isaac-Renton } \text { et al. [62]; } \\
\text { Betancourt \& Rose, } \\
\text { [45]; Budu-Amoako } \\
\text { et al. }[46,47]\end{array}$ \\
\hline$\%$ Infectious & $\begin{array}{l}\text { Point estimate } \\
(50 \%)\end{array}$ & $\begin{array}{l}\text { It is assumed that not all Cryptosporidium in } \\
\text { groundwater will be of infectious origin based on } \\
\text { findings for surface water (Table 2). It is estimated } \\
\text { that the proportion in groundwater may be higher } \\
\text { than surface water due to septic tank inputs. } \\
\text { Therefore, } 50 \% \text { was chosen, guided by informal } \\
\text { expert advice }\end{array}$ & \\
\hline $\begin{array}{l}\text { Concentration } \\
\text { (oocysts/l) }\end{array}$ & $\begin{array}{r}\text { Lognormal* } \\
(0 \cdot 02,0 \cdot 09)\end{array}$ & $\begin{array}{l}\text { Data selected from one study that collected } 253 \\
\text { samples from } 149 \text { wells across the United States in } \\
\text { different geographical locations (only study of this } \\
\text { magnitude in North America). Results from other } \\
\text { studies fit within the range reported by Hancock et al. } \\
\text { [48], except for two outlier data points reported by } \\
\text { Budo-Amoako et al. }[46,47 \text { ] of } 7 \cdot 2 \text { and } 8 \cdot 83 \text { oocysts/1 } \\
\text { in agriculturally intensive areas of Prince Edward } \\
\text { Island. Reported median substituted for arithmetic } \\
\text { mean }\end{array}$ & $\begin{array}{l}\text { Hancock et al. }[48] \text {; } \\
\text { Isaac-Renton } \text { et al. [62]; } \\
\text { Betancourt \& Rose, } \\
\text { [45]; Budu-Amoako } \\
\text { et al. }[46,47]\end{array}$ \\
\hline Recovery & $\begin{array}{l}\text { Point estimate } \\
(17 \cdot 5 \%)\end{array}$ & Middle of range reported by Hancock et al. [48] & Hancock et al. [48] \\
\hline \multicolumn{4}{|l|}{ Campylobacter } \\
\hline Prevalence rate & $\begin{array}{l}\text { Point estimate } \\
(1 \cdot 37 \%)\end{array}$ & $\begin{array}{l}\text { US/Canada studies pooled to develop point estimate } \\
\text { (i.e. } 5 / 364 \text { ) }\end{array}$ & $\begin{array}{l}\text { Borchardt et al. [41]; } \\
\text { St. Pierre et al. [63] }\end{array}$ \\
\hline $\begin{array}{l}\text { Concentration } \\
(\mathrm{MPN} / \mathrm{l})\end{array}$ & $\begin{array}{l}\text { Exponential§ } \\
(0 \cdot 28, \text { truncated at } 4)\end{array}$ & $\begin{array}{l}\text { Concentration distribution obtained directly from } \\
\text { New Zealand QMRA study because North } \\
\text { American concentration data for wells were not } \\
\text { available }\end{array}$ & Close et al. [64] \\
\hline Recovery & $\begin{array}{l}\text { Point estimate } \\
(100 \%)\end{array}$ & Conservatively high value & \\
\hline \multicolumn{4}{|l|}{ E. coli $\mathrm{O} 157$} \\
\hline Prevalence rate & $\begin{array}{l}\text { Point estimate } \\
(1 \cdot 9 \%)\end{array}$ & $\begin{array}{l}\text { US/Canadian studies pooled to develop point } \\
\text { estimate (i.e. 7/371) }\end{array}$ & $\begin{array}{l}\text { Borchardt et al. [41]; } \\
\text { Won et al. }[65]\end{array}$ \\
\hline $\begin{array}{l}\text { Concentration } \\
\text { (c.f.u./l) }\end{array}$ & $\begin{array}{l}\text { Uniform } \dagger \\
(0 \cdot 001,0 \cdot 01)\end{array}$ & $\begin{array}{l}\text { Concentration distribution obtained from only US/ } \\
\text { Canadian paper that reports concentration data for } \\
\text { E. coli } \mathrm{O} 157 \text { in groundwater }\end{array}$ & Won et al. $[65]$ \\
\hline Recovery & Point estimate & Conservatively high value & \\
\hline
\end{tabular}


Table 3 (cont.)

\begin{tabular}{|c|c|c|c|}
\hline Model parameter & Distribution & Rationale & Reference \\
\hline \multicolumn{4}{|l|}{ Norovirus } \\
\hline Prevalence rate & $\begin{array}{l}\text { PERT } \$ \\
(0 \cdot 95 \%, 3 \cdot 52 \%, 6 \cdot 25 \%)\end{array}$ & $\begin{array}{l}\text { Contamination rates taken from pooled US/ } \\
\text { Canadian results of all norovirus samples. Min = } \\
\text { lowest (sample) \% occurrence [66]; most likely = \% of } \\
\text { samples from pooled studies for norovirus (excluding } \\
\text { Borchardt } \text { et al. [67] GII data); max = highest sample } \\
\% \text { occurrence [68] }\end{array}$ & $\begin{array}{l}\text { Abbazadegan et al. } \\
\text { [66]; Borchardt et al. } \\
\text { [68]; Locas et al. [69]; } \\
\text { Hunt et al. [70]; } \\
\text { Borchardt et al. [67]; } \\
\text { Allen [71] }\end{array}$ \\
\hline $\begin{array}{l}\text { Concentration } \\
(\mathrm{gc} / \mathrm{l})\end{array}$ & $\begin{array}{l}\text { Weibull\| } \\
(0 \cdot 6267,23 \cdot 72)\end{array}$ & $\begin{array}{l}\text { Fit to raw untreated groundwater data for norovirus } \\
\text { GI obtained from Dr Borchardt ( } 2010 \text { data) }\end{array}$ & Unpublished data \\
\hline Recovery & $\begin{array}{l}\text { Point estimate } \\
(100 \%)\end{array}$ & Conservatively high value & \\
\hline
\end{tabular}

c.f.u., Colony-forming units; gc, genomic copies; MPN, most probable number; QMRA, quantitative microbial risk assessment.

* Lognormal distribution (arithmetic mean, standard deviation).

$\dagger$ Uniform distribution (minimum, maximum).

$\$$ PERT distribution (minimum, most likely, maximum).

$\S$ Exponential distribution (scale parameter).

\| Weibull distribution (shape parameter, scale parameter).

(4) Chemical disinfection (ozone, chlorine, chlorine dioxide, or chloramines) with/without coagulation/ flocculation/sedimentation, but no filtration or UV disinfection.

(5) UV and chemical disinfection (with/without other treatment).

Specific log removal/inactivation ranges were developed using the minimum and maximum levels of treatment that could be achieved for each pathogen and each of the original 19 treatment categories and source water types (ground vs. surface). These values were developed from literature references compiled by Health Canada for various treatment types for five reference pathogens: Giardia, Cryptosporidium, rotavirus (used as a proxy for norovirus treatment in this QMRA), Campylobacter and E. coli (based on studies used for the Health Canada QMRA model) [34]. As no single virus has all the characteristics of an ideal reference virus, this risk assessment incorporates the key characteristics of rotavirus, with $\mathrm{CT}$ values based on hepatitis A virus (HAV) and poliovirus [United States Environmental Protection Agency (USEPA, 1999)] as the best currently available disinfection information for enteric viruses commonly found in surface water and groundwater sources (Health Canada, 2011). For each of the five treatment categories above, the log-reduction ranges were combined and PERT log removal distributions were developed such that each source water type, treatment category, and pathogen has a unique distribution.
Mode values are based upon population-weighted fitting (sampling from population size/treatment level combinations for each pathogen/treatment combination) (Supplementary Table S5). Applying a PERT distribution is less sensitive to the extremes of the distribution than a triangular distribution and more sensitive to the most likely value (mode) [17].

Previously published dose-response models for each of the five pathogens were obtained from the peerreviewed literature (Table 5). The morbidity factor $\left(P_{\text {ill/inf }}\right)$ for each organism is presented in Table 5. These values were based on an in-depth literature review conducted by USEPA [22].

\section{RESULTS}

Estimated daily and annual probabilities of infection, numbers of cases of infection/year and the corresponding cases of illness/year for all five pathogens for all three water supplies are presented in Tables 6 , 7, and Supplementary Tables S6-S8.

\section{Private wells}

QMRA models estimated a total of 78073 cases of illness/ year (90\% PI 38 466-128 109) resulting from consumption of water containing Giardia, Cryptosporidium, Campylobacter, E. coli $\mathrm{O} 157$ and norovirus from untreated private wells in Canada (Supplementary Table S6). This corresponds to $\sim 0.027$ AGI cases/ 
Table 4. Surface water pathogen inputs selected for use in the small systems QMRA models for Giardia, Cryptosporidium, Campylobacter, E. coli O157 and norovirus

\begin{tabular}{|c|c|c|c|}
\hline Model parameter & Distribution & Rationale & Reference \\
\hline \multicolumn{4}{|l|}{ Giardia } \\
\hline Prevalence rate & $\begin{array}{l}\text { PERT* } \\
(0 \%, 19 \%, 42 \%)\end{array}$ & $\begin{array}{l}\text { Selected range published by Wilkes et al. [72] because } \\
\text { study captured range of prevalence in different } \\
\text { seasons. }\end{array}$ & Wilkes et al. [72] \\
\hline $\begin{array}{l}\text { Concentration } \\
\text { (cysts/l) }\end{array}$ & $\begin{array}{l}\text { Lognormal } \uparrow \\
(0 \cdot 176,2 \cdot 14)\end{array}$ & $\begin{array}{l}\text { Health Canada data from multiple source waters were } \\
\text { fit to lognormal distribution. Reported median } \\
\text { substituted for arithmetic mean }\end{array}$ & $\begin{array}{l}\text { Unpublished Health } \\
\text { Canada data; Wilkes et al. } \\
\text { [73]; Payment et al. [74] }\end{array}$ \\
\hline Recovery & - & Health Canada data were adjusted for recovery & $\begin{array}{l}\text { Unpublished Health } \\
\text { Canada data }\end{array}$ \\
\hline \multicolumn{4}{|l|}{ Cryptosporidium } \\
\hline Prevalence rate & $\begin{array}{l}\text { PERT* } \\
(0 \%, 17 \%, 72 \%)\end{array}$ & $\begin{array}{l}\text { Selected range published by Wilkes et al. }[72] \text {, } \\
\text { reflecting prevalence across different seasons. Range } \\
\text { compares to values published by a second Canadian } \\
\text { surface water study [75] }\end{array}$ & $\begin{array}{l}\text { Wilkes et al. [72]; Ruecker } \\
\text { et al. [75] }\end{array}$ \\
\hline$\%$ Infectious & $\begin{array}{l}\text { PERT* } \\
\quad(0 \%, 4 \cdot 1 \%, 8 \%)\end{array}$ & $\begin{array}{l}\text { Based on } \% \text { samples positive for } C \text {. hominis, } \\
\text { C. parvum. Min, } 0 \% \text {; most likely (mean of all studies, } \\
8 \%, 1 \cdot 6 \%, 2 \cdot 7 \%) \text {; } \max (8 \%) \text { from Pintar } \text { et al. }[76]\end{array}$ & $\begin{array}{l}\text { Pintar et al. [76]; Ruecker } \\
\text { et al. [75]; Wilkes et al. [39] }\end{array}$ \\
\hline $\begin{array}{l}\text { Concentration } \\
\text { (oocyst/l) }\end{array}$ & $\begin{array}{l}\text { Lognormal } \uparrow \\
(0 \cdot 0857,0 \cdot 4269)\end{array}$ & $\begin{array}{l}\text { Health Canada data from multiple source waters were } \\
\text { fit to lognormal distribution. }\end{array}$ & $\begin{array}{l}\text { Unpublished Health } \\
\text { Canada data; Wilkes et al. } \\
\text { [73]; Payment et al. [74]; } \\
\text { Ruecker et al. [77] }\end{array}$ \\
\hline Recovery & - & Health Canada data were adjusted for recovery & $\begin{array}{l}\text { Unpublished Health } \\
\text { Canada data }\end{array}$ \\
\hline \multicolumn{4}{|l|}{ Campylobacter } \\
\hline Prevalence rate & $\begin{array}{l}\text { PERT }^{*} \\
(0 \%, 25 \%, 57 \cdot 8 \%)\end{array}$ & $\begin{array}{l}0 \% \text { is the lowest seasonal prevalence rate; } 25 \% \text { is the } \\
\text { overall prevalence rate from Wilkes et al. }[73] ; 57 \cdot 8 \% \text { is } \\
\text { the largest seasonal prevalence rate }\end{array}$ & $\begin{array}{l}\text { St Pierre et al. [63]; Wilkes } \\
\text { et al. }[72,73] \text {; }\end{array}$ \\
\hline $\begin{array}{l}\text { Concentration } \\
\text { (MPN/l) }\end{array}$ & $\begin{array}{l}\text { Lognormal } \dagger \\
(3 \cdot 04,255)\end{array}$ & $\begin{array}{l}\text { Data from one Canadian study [63] and MPN data } \\
\text { from FoodNet Canada were fit to a lognormal } \\
\text { distribution }\end{array}$ & $\begin{array}{l}\text { St Pierre et al. }[63] \\
\text { FoodNet Canada [78] }\end{array}$ \\
\hline Recovery & $\begin{array}{l}\text { Point estimate } \\
(100 \%)\end{array}$ & Conservatively high value & \\
\hline \multicolumn{4}{|l|}{ E. coli $\mathrm{O} 157$} \\
\hline Prevalence rate & $\begin{array}{l}\text { PERT* } \\
\quad(0 \%, 1 \%, 2 \cdot 3 \%)\end{array}$ & $\begin{array}{l}\text { Low, most likely and high values selected from pooled } \\
\text { literature }\end{array}$ & $\begin{array}{l}\text { Johnson et al. [79]; Wilkes } \\
\text { et al. [72, 73]; Jokinen et al. } \\
\text { [80] }\end{array}$ \\
\hline $\begin{array}{l}\text { Concentration } \\
\text { (c.f.u./l) }\end{array}$ & $\begin{array}{l}\text { Uniform } \$ \\
(0 \cdot 001,0 \cdot 01)\end{array}$ & $\begin{array}{l}\text { Used same values as Won et al. [65] paper } \\
\text { (groundwater) }\end{array}$ & Won et al. [65] \\
\hline Recovery & $\begin{array}{l}\text { Point estimate } \\
(100 \%)\end{array}$ & Conservatively high value & \\
\hline \multicolumn{4}{|l|}{ Norovirus } \\
\hline Prevalence rate & $\begin{array}{l}\text { PERT* } \\
(0 \%, 6 \cdot 3 \%, 10 \%)\end{array}$ & $\begin{array}{l}\text { Data from freshwater recreational waters in Europe } \\
\text { and Canadian rivers }\end{array}$ & $\begin{array}{l}\text { Wyn-Jones et al. [40]; } \\
\text { Wilkes et al. [39] }\end{array}$ \\
\hline $\begin{array}{l}\text { Concentration } \\
(\mathrm{gc} / \mathrm{l})\end{array}$ & $\begin{array}{l}\text { PERT* } \\
(1,10,400)\end{array}$ & $\begin{array}{l}\text { Only North American study that reported } \\
\text { concentrations in } \mathrm{gc} / 1\end{array}$ & Corsi et al. $[81]$ \\
\hline Recovery & $\begin{array}{l}\text { Point estimate } \\
(100 \%)\end{array}$ & Conservatively high value & \\
\hline
\end{tabular}

c.f.u., Colony-forming units; gc, genomic copies; MPN, most probable number; QMRA, quantitative microbial risk assessment.

* PERT distribution (minimum, most likely, maximum) - expert judgement informed the choice of minimum, mode and maximum values when the literature were sparse (for example, a minimum value of $0 \%$ was included based on our understanding that samples could be negative for this pathogen).

$\dagger$ Lognormal distribution (arithmetic mean, standard deviation).

\$ Uniform distribution (minimum, maximum). 
Table 5. Dose-response functions and morbidity values selected for the Giardia, Cryptosporidium, Campylobacter, E. coli O157 and norovirus models

\begin{tabular}{lll}
\hline \hline Pathogen & Dose response and morbidity & Reference \\
\hline Giardia & Exponential, $r=0 \cdot 0199$ & Rose et al. [82] \\
& $P_{\text {ill/inf }}=$ Uniform distribution $(0 \cdot 2-0 \cdot 7)$ & USEPA [22] \\
Cryptosporidium & Exponential, $r=0 \cdot 018$ & Messner et al. [83] \\
& $P_{\text {ill/inf }}=$ Uniform distribution $(0 \cdot 2-0 \cdot 7)$ & USEPA [22] \\
Campylobacter & Exact beta-Poisson $(\alpha=0 \cdot 1453, \beta=8 \cdot 007)$ & Schmidt et al. [84] \\
& $P_{\text {ill/inf }}=$ Uniform distribution $(0 \cdot 1-0 \cdot 6)$ & USEPA [22] \\
E. coli O157 & Approx. beta-Poisson $(\alpha=0 \cdot 4, \beta=37 \cdot 6)$ & Median of 10000 iterations by Teunis et al. [85]; \\
& $P_{\text {ill/inf }}=$ Uniform distribution $(0 \cdot 2-0 \cdot 6)$ & Bielaszewska et al. [86] USEPA, [22] \\
Norovirus* & Exact beta-Poisson $(\alpha=0 \cdot 04, \beta=0 \cdot 055)$ & Teunis et al. [87] USEPA [21] \\
& $P_{\text {ill/inf }}=$ Uniform distribution $(0 \cdot 3-0 \cdot 8)$ & \\
\hline \hline
\end{tabular}

* It was assumed that the entire population lacks immunity to norovirus.

person-year ( $\mathrm{p}-\mathrm{yr}$ ) for those consuming water from these supplies. Norovirus is estimated to be responsible for $71 \cdot 2 \%$ of symptomatic cases $(n=55558)$, followed by Campylobacter $(n=9273, \quad 11.9 \%)$, Cryptosporidium ( $n=11398,14 \cdot 6 \%)$, Giardia (1207, $1 \cdot 53 \%)$ and E. coli $\mathrm{O} 157$ (637, 0.82\%).

\section{Small groundwater systems}

The small groundwater systems models predicted that 13035 cases of illness/year (90\% PI 3416-25 698) are attributable to Giardia, Cryptosporidium, Campylobacter, E. coli $\mathrm{O} 157$ and norovirus associated with the consumption of water from small groundwater supplies serving $<1000$ people (Supplementary Table S7). This is an incidence of $\sim 0.016$ AGI cases/p-yr for those consuming water from these supplies. Similar to private wells, norovirus was responsible for the majority of cases $(n=$ $10869,83 \cdot 4 \%)$ followed by Cryptosporidium $(n=1639$, $12 \cdot 6 \%)$, Campylobacter $(n=378,2 \cdot 90 \%)$, Giardia $(n=$ $121,0 \cdot 93 \%)$ and $E$. coli $\mathrm{O} 157(n=28,0 \cdot 21 \%)$.

\section{Small surface water systems}

It is estimated that 12122 cases of illness/year (90\% PI 2974-26274) are attributable to the consumption of water from small Canadian surface water systems (serving $<1000$ people) as a result of Giardia, Cryptosporidium, Campylobacter, E. coli $\mathrm{O} 157$ and norovirus (Supplementary Table S8). This corresponds to an incidence of 0.026 AGI cases/p-yr for those consuming water from these supplies. Norovirus is responsible for the majority of cases of illness $(n=9003$, $74 \cdot 3 \%)$, followed by Giardia $(n=2288,18.9 \%)$, Campylobacter $(n=513,4.23 \%), \quad$ Cryptosporidium $(n=317,2 \cdot 62 \%)$ and E. coli $\mathrm{O} 157(n=1,0 \cdot 008 \%)$.

\section{Waterborne AGI}

In Canada, it is estimated that there are 20.5 million AGI cases that occur annually ( $0 \cdot 6$ cases/p-yr), after accounting for under-diagnosis and under-reporting [1]. This includes both domestically acquired and travel-acquired AGI. Of the total 20.5 million, an estimated 4 million (20\%) domestically acquired cases are attributed to food [1]. The current study estimates that $\sim 103230$ cases $(0 \cdot 003 / \mathrm{p}-\mathrm{yr})$, or $0 \cdot 51 \%$, of the total are attributed to the consumption of water from private wells and small water systems (Table 6).

When comparing cumulative projected AGI incidence rates from all reference pathogens by system type and treatment category, those served by surface water systems with no treatment $(0.098$ cases $/ \mathrm{p}$-yr $)$ or only one treatment barrier, such as chemical disinfection $(0.037$ cases $/ \mathrm{p}$-yr) are more at risk than those served by groundwater systems or private wells (Table 7). Incidence rates for those served by untreated groundwater or private wells were the same $(0.027$ cases/p-yr) and were slightly lower than for those served by groundwater systems treated by chemical disinfection $(0.016$ cases $/ \mathrm{p}$-yr $)$, membrane filtration $(0.008$ cases $/ \mathrm{p}$-yr $)$ or media filtration $(0.012$ cases/ p-yr). The lowest incidence rates were estimated for individuals served by surface water or groundwater sources with both UV and chemical disinfection $(0 \cdot 0005-0 \cdot 001$ cases $/ \mathrm{p}-\mathrm{yr})$.

\section{Sensitivity analysis}

Sensitivity analyses were performed to determine model input contributions to each model (Supplementary Table S4). Pathogen concentration was predictably correlated with estimated numbers of illnesses, 
Table 6. Estimated total number of domestically acquired Canadian cases of Giardia, Cryptosporidium, Campylobacter, E. coli O157 and norovirus attributable to private wells, small groundwater systems and small surface water systems

\begin{tabular}{|c|c|c|c|c|c|}
\hline Pathogen & $\begin{array}{l}\text { Total estimated } \\
\text { Canadian cases* } \\
(90 \% \mathrm{PI})\end{array}$ & $\begin{array}{l}\text { Projected illnesses } \\
\text { attributable to private } \\
\text { wells }(90 \% \text { PI) }\end{array}$ & $\begin{array}{l}\text { Projected illnesses } \\
\text { attributable to small } \\
\text { GW systems }(90 \% \text { PI) }\end{array}$ & $\begin{array}{l}\text { Projected illnesses } \\
\text { attributable to small } \\
\text { SW systems (90\% PI) }\end{array}$ & $\begin{array}{l}\% \text { of total cases } \\
\text { related to small/ } \\
\text { private systems } \dagger\end{array}$ \\
\hline Giardia & 108507 (70 532-158 740) & $1207(2-7136)$ & $121(0-619)$ & $2288(6-12120)$ & $3 \cdot 33 \%$ \\
\hline Cryptosporidium & $25318(14331-45955)$ & $11398(238-45141)$ & $1639(27-7108)$ & $317(1-1310)$ & $52 \cdot 7 \%$ \\
\hline Campylobacter & 213479 (144 288-308 837) & $9273(1180-19980)$ & $378(45-818)$ & $513(1-1433)$ & $4 \cdot 76 \%$ \\
\hline E. coli $\mathrm{O} 157$ & $16913(6968-29668)$ & $637(124-1528)$ & $28(5-72)$ & $1(0-4)$ & $3 \cdot 94 \%$ \\
\hline Norovirus & $3379990(3002927-3778$ 461) & 55558 (24 323-95 709) & $10869(2211-22736)$ & 9003 (1790-18 930) & $2 \cdot 23 \%$ \\
\hline Total projected cases & & 78073 (38 466-128 109) & $13035(3416-25698)$ & $12122(2974-26274)$ & \\
\hline
\end{tabular}

GW, Groundwater; SW, surface water; PI, Probability interval.

* Data from Public Health Agency of Canada foodborne illness estimates [1]

$\dagger$ Sum of projected illnesses attributable to private wells and small systems divided by total estimated Canadian cases.

Table 7. Comparison of AGI incidence rates by water source type and treatment system category

\begin{tabular}{|c|c|c|c|c|c|c|c|c|c|}
\hline \multirow[b]{2}{*}{ Treatment category } & \multicolumn{3}{|l|}{ Private wells } & \multicolumn{3}{|c|}{ Small groundwater systems } & \multicolumn{3}{|c|}{ Small surface water systems } \\
\hline & $\begin{array}{l}\text { Annual AGI } \\
\text { cases }\end{array}$ & $\begin{array}{l}\text { Population } \\
\text { served }\end{array}$ & Incidence* & $\begin{array}{l}\text { Annual AGI } \\
\text { cases }\end{array}$ & $\begin{array}{l}\text { Population } \\
\text { served }\end{array}$ & Incidence* & $\begin{array}{l}\text { Annual AGI } \\
\text { cases }\end{array}$ & $\begin{array}{l}\text { Population } \\
\text { served }\end{array}$ & Incidence* \\
\hline No treatment & 78073 & 2861602 & $0 \cdot 027$ & 2948 & 108014 & $0 \cdot 027$ & 597 & 6066 & $0 \cdot 098$ \\
\hline Membrane filtration & - & - & - & 365 & 45952 & $0 \cdot 008$ & 788 & 46202 & $0 \cdot 017$ \\
\hline Media filtration & - & - & - & 1991 & 166698 & $0 \cdot 012$ & 3370 & 160789 & $0 \cdot 021$ \\
\hline Chemical disinfection & - & - & - & 7720 & 485244 & $0 \cdot 016$ & 7295 & 197716 & $0 \cdot 037$ \\
\hline $\begin{array}{l}\text { UV and chemical } \\
\text { disinfection }\end{array}$ & - & - & - & 10 & 21111 & $0 \cdot 0005$ & 73 & 64417 & $0 \cdot 011$ \\
\hline
\end{tabular}

AGI, Acute gastrointestinal illness.

* Incidence rate $=$ cases $/ \mathrm{p}-\mathrm{yr}$. 
appearing in the top three factors in all pathogen models with the exception of norovirus $(r=0.442$ $0 \cdot 866$ ), and was the most highly correlated input in both protozoan models. The lack of correlation for norovirus is due to the estimated high exposures for a small fraction of private wells and small systems; for which, the corresponding annual probability of infection is nearly $100 \%$. The prevalence rate was significantly correlated with outputs from the Cryptosporidium, E. coli $\mathrm{O} 157$ and norovirus models $(r=0 \cdot 267-0.755)$. Daily adult water consumption was a significant input in both the Giardia $(r=0 \cdot 187)$ and Campylobacter $(r=0 \cdot 195)$ models.

\section{DISCUSSION}

This study is part of a larger effort to quantify and attribute AGI in Canada to various sources, including drinking water. The objective of the work presented herein is to estimate the number of AGI cases caused by Giardia, Cryptosporidium, Campylobacter, E. coli $\mathrm{O} 157$ and norovirus attributable to the consumption of water from private wells and small community systems in Canada using a QMRA approach.

In Canada, the majority of drinking water systems are small systems. There are significant challenges faced by these systems, which have been identified nationally and internationally as a priority in the drinking water treatment and public health communities. The World Health Organization (WHO) has identified management of small community drinking water supplies as a critical issue for sustainable development and health [23]. The Office of the Inspector General within the USEPA also conducted an evaluation and concluded that effort and resources are needed to help small drinking water systems overcome the challenges to delivering safe drinking water [24]. In Canada, federal, provincial and territorial governments have long recognized the importance of supporting small drinking water systems in the delivery of safe water.

Small drinking water systems face the same issues as larger municipal drinking water systems in the provision of safe water. In 2011, the National Collaborating Centre for Environmental Health (NCCEH) published a report that examined the issue of waterborne disease risks in Canadian small drinking water systems. They found a high proportion of waterborne disease outbreaks occurred in small drinking water systems serving $<5000$ people [25]. While the challenges small systems face are similar to large systems, they are usually operating with fewer people, who have less experience and technical knowledge [26]. Small systems often find it difficult to hire and retain staff with sufficient technical knowledge. In addition, they often have limited time to dedicate to the operation of these systems, as drinking water operators/managers are often responsible for other operations in the community [27]. Distribution system issues exist in small systems too, including inadequate maintenance, high water losses, crossconnections, frequent breaks and slow replacement [25]. Northern communities have unique challenges, due to the presence of permafrost, extreme cold and lack of year-round road networks, as well as difficulty accessing laboratories.

Source protection, adequate treatment and continued monitoring are the tenets of a safe and robust drinking water supply, regardless of size [28]. With small systems, there are more elusive factors associated with the effective operation of a treatment system and delivery of safe water. A recent Canadian study emphasizes the utility of developing water safety plans for small and large water systems and the importance of building community readiness through early engagement [29] to reduce the risk and burden of waterborne illness.

\section{Risk by drinking water source and treatment category}

The combination of source water type (ground vs. surface) and level of drinking water treatment influence the microbial and chemical quality of drinking water. This study illustrates that those individuals served by small surface water systems could be more at risk of AGI than those served by private wells or small groundwater systems (in terms of incidence rates, as shown in Table 7). This study also illustrates that those served by untreated surface water supplies could be at greatest risk for waterborne AGI, although only about 6000 Canadians rely on water from this category. The risk of exposure to waterborne pathogens in small untreated surface water supplies has previously been well documented [12]. Conversely, drinking water systems that treat with UV disinfection are estimated to be at much lower risk for AGI, for both ground and surface water sources. This is attributed to the efficacy of UV disinfection for the inactivation of pathogens in water [30-32].

Small water systems typically have fewer treatment barriers in place compared to larger systems and risk is inherently greater [5, 9]. Small surface water 
supplies that only chlorinate will be more vulnerable to Cryptosporidium and Giardia as these organisms are highly resistant to chlorine; whereas a system with multiple barriers (e.g. coagulation, flocculation, filtration, disinfection) is more effective at removing these protozoa [33, 34].

This work illustrates the efficacy of UV disinfection at inactivating pathogens, reducing the likelihood of AGI cases attributed to drinking water. UV disinfection is highly effective $(>4 \log )$ at inactivating Giardia, Cryptosporidium, Campylobacter, E. coli O157 and norovirus [34]. Although membrane filtration is a robust treatment technology, microfiltration has a nominal pore in a range that will allow viruses to pass through. It is not as effective against rotavirus as UV disinfection [34].

This study also illustrates the impact of private well water quality on the overall burden of waterborne illness in Canada. Engaging private homeowners in the important responsibility of maintaining their wells, treating their drinking water supply, and routinely testing their water is a shared responsibility. Public engagement on the issue of safe well water and stewardship continues to be limited by complacency, inconvenience, cost and privacy concerns, identified consistently by those examining stewardship behaviour of private well owners in Newfoundland [35], Ontario [36], and across Canada [37].

\section{Norovirus}

We estimate between 74\% (private wells) and 83\% (small groundwater systems) of predicted AGI cases are attributed to norovirus. However, the norovirus dose-response relationship and mechanistic interpretation is subject to considerable debate [38] and may need to be revised as improved dose-response information becomes available.

Few published studies have examined the prevalence and concentration of norovirus in Canadian surface water and groundwater systems and this is an identified knowledge gap. The prevalence rate for norovirus in surface waters was based on one Canadian study of rivers [39] and a study of recreational waters in Europe [40]. With respect to groundwater, we recognize that the developed models do not reflect variations in local hydrogeology, which have a significant influence on the transient nature of virus prevalence and concentrations [41].
Norovirus contributes greatly to the total burden of enteric disease in Canada ( 3.4 million cases annually) [1], yet is both under-reported and underdiagnosed, and is not routinely included in national surveillance platforms for enteric illness. In this study, it is estimated that $2 \cdot 23 \%$ of all annual, domestically acquired norovirus cases in Canada could be the result of consumption of water from small or private Canadian water supplies. This is similar to what has been reported in other countries; in the United States, $1.5 \%$ of reported norovirus outbreaks between 2010 and 2012 were linked to waterborne transmission [42]. Similarly, norovirus was found to be the most common pathogen contributing to waterborne disease in groundwater systems in Norway [43]. Future work to consider the impact of secondary transmission in the epidemiology of waterborne norovirus infections could consider the rate of community cases attributed back to these initial illnesses, given its high infectivity.

\section{Cryptosporidium and Giardia}

The developed model predictions that 13354 and 3616 cases of Cryptosporidium and Giardia, respectively, occur in Canada each year from the consumption of untreated or inadequately treated groundwater or surface water from private wells and small water systems. The disease burden for Giardia infections in Canada is high and ranks in the top three reported enteric infections provincially and nationally, with a national incidence rate of 11.43 cases $/ 100000$ people per year [44]. While the cryptosporidiosis disease burden in Canada is lower (1.82 cases/100000 people per year), the number of predicted cases of Cryptosporidium is roughly 9 and 14 times greater in private wells and small groundwater supplies, respectively, than the predicted cases of Giardia in the same systems.

Occurrence studies show that Cryptosporidium oocysts are more prevalent in groundwater sources than Giardia cysts [45-48], primarily due to their smaller size, which allows for more rapid downward ingress through overburden layers [49]. Moreover, Cryptosporidium oocysts are more resistant to low subsurface temperatures and thus persist in the environment for longer periods of time [50]. In addition, Cryptosporidium is more resistant to chemical disinfection than Giardia [51]. Many private or small system groundwater sources are actually undocumented GUDI (groundwater under the direct influence of surface water), which explains the reported presence of 
oocysts/cysts in these waters [52]. In fact, the presence of large diameter pathogens such as Cryptosporidium or Giardia in groundwater is sometimes used to define a GUDI water source [53].

\section{Campylobacter}

Campylobacter is the leading cause of bacterial gastrointestinal illness in Canada, with an incidence of $29 \cdot 3$ cases $/ 100000$ people per year in 2012 [44]. In this study, we estimate that Campylobacter is the third leading cause of waterborne AGI in private well and small water system users in Canada. This represents an estimated $4 \cdot 76 \%$ of an estimated 213000 domestically acquired cases of Campylobacter that occur annually in Canada, after accounting for under-reporting and under-diagnosis [1]. A recent source attribution study of campylobacteriosis using a systematic review and meta-analysis framework demonstrated that untreated drinking water is a significant risk factor [54]. Previous Canadian studies have demonstrated that private wells are a risk factor for campylobacteriosis [55].

\section{E. coli 0157}

The E. coli O157 QMRA model estimated that 666 cases were attributable to the consumption of untreated or inadequately treated water from private wells, small surface water or small groundwater supplies. In Canada, the incidence rate for verotoxigenic E. coli (VTEC) is 1.81 cases $/ 100000$ people per year based on reported cases [44]. In a review of E. coli $\mathrm{O} 157$ outbreaks 1982-2002 in the United States, $15 \%$ of all outbreak cases were linked to the consumption of drinking water [56]. Similarly, a source attribution study of E. coli $\mathrm{O} 157$ infections in the United States found that $5 \%$ of sporadic infections and $73 \%$ of outbreak infections were associated with consumption of untreated water [57]. In Canada, private well water and small system supplies are recognized risks of $E$. coli O157 infection $[58,59]$.

\section{Study limitations/model uncertainty}

These models rely on data from the literature and some Canadian surveillance systems, and thus are not site-specific and do not reflect the influence of local hydrogeology, as well as temporal and spatial differences specific to local systems. Rather, the models are designed to reflect the range of likely scenarios for small water systems and private wells in Canada.
The inputs are designed to encompass both site-to-site variability and uncertainty in the enteric pathogen concentration and prevalence rates of Canadian groundwater and surface water supplies. The models are limited by data availability. Where pathogen data were available, few studies documented pathogen concentrations in the source water; only 10 of 22 North American studies of Campylobacter, E. coli O157 and norovirus documented concentrations, of which two reported only zero values/non-detects, and the remaining reported presence/absence results (Supplementary Tables S1, S2). While we attempted to capture the influence of seasonality on input distributions, by including multi-year studies, these data were not always available. The concentration and prevalence rate (occurrence) of pathogens in Canadian source waters, collected over multiple years, are crucial data needed to refine future burden estimates.

Dose-response models continue to be a limitation of all QMRA models [60]. Additionally, the ratios for the probability of illness given infection do not account for variations we would expect in various sub-populations or the dependence of morbidity upon dose [61]. The uncertainty around the infectivity of different strains and genotypes of an organism, as well as the large variability in host/pathogen interactions, contributes to the uncertainty in the outputs. Future knowledge gaps that could be explored include: (1) rigorous reporting of outbreak details (attack rates for different subtypes of organisms), and (2) community aetiology studies to examine the prevalence of asymptomatic carriage of pathogens.

Deriving a burden estimate by focusing on specific (reference) pathogens may under-estimate illness. There is the potential that there are additional AGI cases attributable to other pathogens, such as Shigella, Salmonella, and other enteric viruses [10]. The continued development and optimization of (clinical and environmental) pathogen detection methods will help to further inform which pathogens are most often associated with AGI, and refine our estimate. Despite these inherent limitations, the five pathogens that were used are recognized waterborne reference pathogens (for both the WHO and Health Canada drinking water guidelines) and thus, are considered to adequately represent the majority of known microbial risks from these water supplies. Another consideration that was not explored in this analysis but could influence our understanding of risk is the role that socioeconomic factors play in the burden of waterborne disease, both in urban and rural areas. 
Finally, it is important to note that this study did not consider waterborne illness from recreational water, or water for other uses such as irrigation, medical uses, or building water systems. Other types of waterborne illness, such as respiratory illnesses, eye, ear or skin infections, and wound infections were also not considered. Future iterations of this work could assess the burden of the broader spectrum of waterborne diseases, to inform the appropriate allocation of resources and public health interventions.

\section{CONCLUSIONS}

Based on QMRA estimates, the consumption of untreated private well water or inadequately treated water from small surface water and groundwater supplies in Canada may be responsible for an estimated 103230 AGI cases annually (90\% PI 52 419-166 286), representing a small proportion of the burden when considering other routes of transmission. By pathogen, waterborne AGI is attributed to norovirus (73\%), Cryptosporidium (13\%), Campylobacter (10\%), Giardia (3.5\%), and E. coli $\mathrm{O} 157$ $(0 \cdot 6 \%)$ in this study.

The results indicate that those served by small surface water systems in Canada with no treatment or inadequate treatment (e.g. chemical disinfection only) are more at risk for AGI than those served by untreated or inadequately treated groundwater supplies. Private wells and small water systems on both surface and groundwater systems are potential sources of parasitic, viral and bacterial infections. Previous Canadian studies focusing on waterborne outbreaks have made similar conclusions $[9,10]$. The results continue to illustrate the efficacy of water safety interventions, including the combined use of physical removal with UV and chemical disinfection, for small and private systems, regardless of water source, to reduce public health risk and AGI rates.

Small drinking water systems face the same issues as larger systems with respect to producing potable water; however, they typically have fewer resources, experience and technical knowledge. This is a significant challenge when faced with additional operational objectives, such as implementing a multiple barrier approach based on risk assessment and risk management. The key strength of multi-barrier systems is that the limitations or failure of one or more barriers may be compensated by the effective operation of the remaining barriers [88]. A multiple barrier approach requires an understanding of the hazards, an assessment of the potential risks through the entire drinking water system, and resources to implement a management plan to address those risks. New research focusing on the importance of community engagement in the delivery of safe water, and the utility of water safety plans [29] may help to inform next steps for communities relying on small water systems. Engaging private well owners in the stewardship of their wells is an ongoing responsibility shared by private well owners and governments at all levels, and can be facilitated by effective knowledge translation strategies to inform Canadians about known risks and effective treatment technologies to reduce risk.

In addition to providing a mechanism to assess the potential burden of AGI attributed to small systems and private well water in Canada, this research demonstrates that QMRA is an effective source attribution tool when there is a lack of randomized controlled trial data to evaluate the public health risk of an exposure source. QMRA is also a powerful tool for identifying existing knowledge gaps on the national scale to inform future surveillance and research efforts.

\section{SUPPLEMENTARY MATERIAL}

For supplementary material accompanying this paper visit http://dx.doi.org/10.1017/S0950268815002071.

\section{ACKNOWLEDGEMENTS}

We acknowledge Dr Mark Borchardt and the research team of the Wisconsin WAHTER Study (Water and Health Trial for Enteric Risks) and Dr Beth Parker and the Ontario Ministry of Research and Innovation Ontario Research Fund (Research Excellence Round 3 Project no. 03-061) for their contribution to the norovirus datasets used in this publication. We also acknowledge the contributions of Dr Michael Messner US EPA, Dr Michael Beach US CDC, and Dr Julia Gargano US CDC.

\section{DECLARATION OF INTEREST}

None.

\section{REFERENCES}

1. Thomas MK, et al. Estimates of the burden of foodborne illness in Canada for 30 specified pathogens and 
unspecified agents, circa 2006. Foodborne Pathogens and Disease 2013; 10: 639-648.

2. Statistics Canada. Households and the environment survey (HES) 2011. 2013; 3881.

3. Jones AQ, et al. Public perceptions of drinking water: a postal survey of residents with private water supplies. BMC Public Health 2006; 6: 94-94.

4. Health Canada. What's in your well? - a guide to well water treatment and maintenance (http://www.hc-sc.gc. ca/ewh-semt/pubs/water-eau/well-puits-eng.php).

5. Statistics Canada. Survey of drinking water plants 2011. Published May 2013, Ministry of Industry, Statistics Canada. Available at: http://www.statcan.gc.ca/pub/16403-x/16-403-x2013001-eng.pdf.

6. Locas A, et al. Groundwater microbiological quality in Canadian drinking water municipal wells. Canadian Journal of Microbiology 2008; 54: 472-478.

7. Uhlmann S, et al. Where's the pump? Associating sporadic enteric disease with drinking water using a geographic information system, in British Columbia, Canada, 1996-2005. Journal of Water and Health 2009; 7: 692-698.

8. Charrois JWA. Private drinking water supplies: challenges for public health. Canadian Medical Association Journal 2010; 182: 1061-1064.

9. Hrudey SE, Hrudey EJ. Safe Drinking Water: Lessons from Recent Outbreaks in Affluent Nations, 2004, IWA Publishing, London UK, pp. 409.

10. Schuster CJ, et al. Infectious disease outbreaks related to drinking water in Canada, 1974-2001. Canadian Journal of Public Health 2005; 96: 254-258.

11. MICRORISK. Intestinal illness through drinking water in Europe, MicroRisk (Microbiological risk assessment: a scientific basis for managing drinking water safety from source to tap) (http://www.microrisk.com/ uploads/microrisk_intestinal_illness_through_drinking_ water.pdf). Accessed 4 November 2009.

12. Craun MF, et al. Waterborne outbreaks reported in the United States. Journal of Water and Health 2006; 4 (Suppl. 2): 19-30.

13. Murphy HM, et al. Estimating the number of cases of acute gastrointestinal illness (AGI) associated with Canadian municipal drinking water systems. Epidemiology and Infection. doi:10.1017/S0950268815002083.

14. Haas CN, Rose JB, Gerba CP. Quantitative Microbial Risk Assessment. New York, NY: John Wiley \& Sons, 1999, pp. 426-429.

15. Howard G, Pedley S, Tibatemwa S. Quantitative microbial risk assessment to estimate health risks attributable to water supply: can the technique be applied in developing countries with limited data? Journal of Water and Health 2006; 4: 49-65.

16. Murphy HM, et al. A systematic review of waterborne disease burden methodologies from developed countries. Journal of Water and Health 2014; 12: 634 655.

17. Vose D. Risk Analysis: A Quantitative Guide, 3rd edn. West Sussex, UK. John Wiley \& Sons, 2008.

18. 2011 Census of Canada. (http://www.statcan.gc.ca/pub/ 91-215-x/2013002/part-partie1-eng.htm).
19. Pintar KDM, et al. Water consumption habits of a south-western Ontario community. Journal of Water and Health 2009; 7: 276.

20. Roseberry AM, Burmaster DE. Lognormal distributions for water intake by children and adults. Risk Analysis 1992; 12: 99-104.

21. Hynds PD, Thomas MK, Pintar KDM. Contamination of groundwater systems in the US and Canada by enteric pathogens, 1990-2013: a review and pooled-analysis. PLoS ONE 2014; 9: e93301.

22. USEPA. Quantitative microbial risk assessment to estimate illness in freshwater impacted by agricultural animal sources of fecal prevalence, 2010. EPA 822-R-10-005.

23. WHO. Guidelines for safe drinking water, 4th edn. 2011, World Health Organisation, (http://apps.who.int/iris/bitstream/10665/44584/1/9789241548151_eng.pdf).

24. USEPA. Much effort and resources needed to help small drinking water systems overcome challenges, 2006. 2006-P-00026.

25. National Collaborating Centres for Public Health. Water-borne disease outbreaks in Canadian small drinking water systems (http://www.nccph.ca/docs/ SDWS_Water-borne_EN.pdf). Accessed 5 September 2014.

26. Pons W, et al. Experience, training and confidence among small, non-community drinking water system operators in Ontario. Journal of Water and Health 2014; 12: 782-790.

27. USEPA. Water: small systems and capacity development (http://water.epa.gov/type/drink/pws/smallsystems/ basicinformation.cfm). Accessed 5 September 2014.

28. Huck PM, Coffey BM. The importance of robustness in drinking-water systems. Journal of Toxicology and Environmental Health, Part A 2004; 67: 1581-1590.

29. Kot M, Castleden H, Gagnon GA. The human dimension of water safety plans: a critical review of literature and information gaps. Environmental Reviews 2015; 23: 24-29.

30. Craik SA, et al. Inactivation of Giardia muris cysts using medium-pressure ultraviolet radiation in filtered drinking water. Water Research 2000; 34: 4325-4332.

31. Craik SA, et al. Inactivation of Cryptosporidium parvum oocysts using medium- and low-pressure ultraviolet radiation. Water research 2001; 35: 1387-1398.

32. Hijnen WAM, Beerendonk EF, Medema GJ. Inactivation credit of UV radiation for viruses, bacteria and protozoan (oo)cysts in water: a review. Water Research 2006; 40: 3-22.

33. Betancourt WQ, Rose JB. Drinking water treatment processes for removal of Cryptosporidium and Giardia. Veterinary Parasitology 2004; 126: 219-234.

34. Douglas I, et al. QMRA - Probabilistic model to estimate annual risk of illness and DALYs based on source water pathogens and treatment barriers. Version 15-05FINS 2015.

35. Roche S, et al. Investigating public perceptions and knowledge translation priorities to improve water safety for residents with private water supplies: a crosssectional study in Newfoundland and Labrador. BMC Public Health 2013; 13: 1-13. 
36. Hexemer AM, et al. An investigation of bacteriological and chemical water quality and the barriers to private well water sampling in a Southwestern Ontario community. Journal of Water and Health 2008; 6: 521-525.

37. Kreutzwiser R, et al. Understanding stewardship behaviour: factors facilitating and constraining private water well stewardship. Journal of Environmental Management 2011; 92: 1104-1114.

38. Schmidt PJ. Norovirus dose-response: are currently available data informative enough to determine how susceptible humans are to infection from a single virus? Risk Analysis 2015; 35: 1364-1383.

39. Wilkes G, et al. Bacteria, viruses, and parasites in an intermittent stream protected from and exposed to pasturing cattle: prevalence, densities, and quantitative microbial risk assessment. Water Research 2013; 47: 6244-6257.

40. Wyn-Jones AP, et al. Surveillance of adenoviruses and noroviruses in European recreational waters. Water Research 2011; 45: 1025-1038.

41. Borchardt MA, et al. Incidence of enteric viruses in groundwater from household wells in Wisconsin. Applied and Environmental Microbiology 2003; 69: 1172-1180.

42. Leshem E, et al. Genotype GI.6 Norovirus, United States, 2010-2012. Emerging Infectious Diseases 2013; 19(8).

43. Kvitsand HM, Fiksdal L. Waterborne disease in Norway: emphasizing outbreaks in groundwater systems. Water Science and Technology 2010; 61: 563-571.

44. PHAC. Public Health Agency Table, 2009-2011: national number and rate (per 100000 population per 100000 live births) of reported cases by age group and sex (http://dsol-smed.phac-aspc.gc.ca/dsol-smed/ndis/ index-eng.php).

45. Betancourt WQ, Rose JB. Microbiological assessment of ambient waters and proposed water sources for restoration of a Florida wetland. Journal of Water and Health 2005; 3: 89-100.

46. Budu-Amoako E, et al. Giardia and Cryptosporidium on dairy farms and the role these farms may play in contaminating water sources in Prince Edward Island, Canada. Journal of Veterinary Internal Medicine 2012; 26: $668-673$.

47. Budu-Amoako E, et al. Occurrence of Cryptosporidium and Giardia on beef farms and water sources within the vicinity of the farms on Prince Edward Island, Canada. Veterinary Parasitology 2012; 184: 1-9.

48. Hancock CM, Rose JB, Callahan M. Cryptosporidium and Giardia in US groundwater. Journal of the American Water Works Association 1998; 90: 58-61.

49. Solo-Gabriele HM, et al. Occurrence of Cryptosporidium oocysts and Giardia cysts in water supplies of San Pedro Sula, Honduras. Revista Panamericana de Salud Pública 1998; 4: 398-400.

50. Carmena D. Waterborne transmission of Cryptosporidium and Giardia: detection, surveillance and implications for public health. In: Mendez-Vilas A, ed. Current Research, Technology and Education Topics in Applied Microbiology and Microbial Biotechnology, Volume 1. Badajoz, Spain: Formatex, 2010.
51. Korich DG, et al. Effects of ozone, chlorine dioxide, chlorine, and monochloramine on Cryptosporidium parvum oocyst viability. Applied and Environmental Microbiology 1990; 56: 1423-1428.

52. Council of Canadian Academies. The sustainable management of groundwater in Canada: The Expert Panel on Groundwater (http://www.scienceadvice.ca/en/ assessments/completed/groundwater.aspx) 2009.

53. Environmental Science and Program Management Division. Appendix A. Protocol for determining groundwater under the direct influence of surface water (https:// www.novascotia.ca/nse/water/docs/Treatment_Standards_ for_Municipal_Drinking_Water_Systems_Appendix \%20A. pdf). Accessed 7 July 2014.

54. Domingues AR, et al. Source attribution of human campylobacteriosis using a meta-analysis of case-control studies of sporadic infections. Epidemiology and Infection 2012; 140: 970-981.

55. Lévesque $\mathbf{S}$, et al. Campylobacteriosis in urban versus rural areas: a case-case study integrated with molecular typing to validate risk factors and to attribute sources of infection. PLoS ONE 2013; 8: e83731.

56. Rangel JM, et al. Epidemiology of Escherichia coli O157:H7 outbreaks, United States, 1982-2002. Emerging Infectious Diseases 2004; 11: 603-609.

57. Cole D, et al. Attributing sporadic and outbreakassociated infections to sources: blending epidemiological data. Epidemiology and Infection 2014; 142: 295-302.

58. Hrudey SJ, et al. A fatal waterborne disease epidemic in Walkerton, Ontario: comparison with other waterborne outbreaks in the developed world. Water Science and Technology 2004; 47: 7-14.

59. Michel P, et al. Temporal and geographical distributions of reported cases of Escherichia coli O157:H7 infection in Ontario. Epidemiology and Infection 1999; 122: 193-200.

60. Havelaar AH, Swart AN. Impact of acquired immunity and dose-dependent probability of illness on quantitative microbial risk assessment. Risk Analysis 2014; 34: 1807-1819.

61. Gerba CP, Rose JB, Haas CN. Sensitive populations: who is at the greatest risk? International Journal of Food Microbiology 1996; 30: 113-23.

62. Isaac-Renton J, et al. Epidemic and endemic seroprevalence of antibodies to Cryptosporidium and Giardia in residents of three communities with different drinking water supplies. American Journal of Tropical Medicine and Hygiene 1999; 60: 578-583.

63. St Pierre K, et al. Thermotolerant coliforms are not a good surrogate for Campylobacter spp. in environmental water. Applied and Environmental Microbiology 2009; 75: 6736-6744.

64. Close M, et al. Microbial groundwater quality and its health implications for a border-strip irrigated dairy farm catchment, South Island, New Zealand. Journal of Water and Health 2008; 6: 83-98.

65. Won H, Gill A, LeJeune JT. Microbial quality and bacteria pathogens in private wells used for drinking water in northeastern Ohio. Journal of Water and Health 2013; 11: 555. 
66. Abbaszadegan M, LeChevallier M, Gerba C. Occurrence of viruses in US groundwaters. Journal of the American Water Works Association 2003; 95: 107-120.

67. Borchardt MA, et al. Viruses in nondisinfected drinking water from municipal wells and community incidence of acute gastrointestinal illness. Environmental Health Perspectives 2012; 120: 1272-1279.

68. Borchardt MA, Haas NL, Hunt RJ. Vulnerability of drinking-water wells in La Crosse, Wisconsin, to enteric-virus contamination from surface water contributions. Applied and Environmental Microbiology 2004; 70: 5937-5946.

69. Locas A, et al. Virus occurrence in municipal groundwater sources in Quebec, Canada. Canadian Journal of Microbiology 2007; 53: 688-694.

70. Hunt RJ, et al. Assessment of sewer source contamination of drinking water wells using tracers and human enteric viruses. Environmental Science and Technology 2010; 44: 7956-7963.

71. Allen AS. Vulnerability of a fractured bedrock aquifer to emerging sewage-derived contaminants and their use as indicators of virus contamination (thesis), 2013.

72. Wilkes G, et al. Seasonal relationships among indicator bacteria, pathogenic bacteria, Cryptosporidium oocysts, Giardia cysts, and hydrological indices for surface waters within an agricultural landscape. Water Research 2009; 43: 2209-2223.

73. Wilkes G, et al. Associations among pathogenic bacteria, parasites, and environmental and land use factors in multiple mixed-use watersheds. Water Research 2011; 45: 5807-5825.

74. Payment P, et al. Occurrence of pathogenic microorganisms in the Saint Lawrence River (Canada) and comparison of health risks for populations using it as their source of drinking water. Canadian Journal of Microbiology 2000; 46: 565-576.

75. Ruecker NJ, et al. Molecular and phylogenetic approaches for assessing sources of Cryptosporidium contamination in water. Water Research 2012; 46: 5135-5150.

76. Pintar KD, et al. Considering the risk of infection by Cryptosporidium via consumption of municipally treated drinking water from a surface water source in a Southwestern Ontario community. Risk Analysis 2012; 32: $1122-1138$.
77. Ruecker NJ, et al. Tracking host sources of Cryptosporidium spp. in raw water for improved health risk assessment. Applied and Environmental Microbiology 2007; 73: 3945-3957.

78. FoodNet Canada. Unpublished surface water surveillance data (contact katarina.pintar@phac-aspc.gc.ca), 2013.

79. Johnson JY, et al. Prevalence of Escherichia coli O157: H7 and Salmonella spp. in surface waters of southern Alberta and its relation to manure sources. Canadian Journal of Microbiology 2003; 49: 326-335.

80. Jokinen C, et al. Molecular subtypes of Campylobacter spp., Salmonella enterica, and Escherichia coli O157:H7 isolated from faecal and surface water samples in the Oldman River watershed, Alberta, Canada. Water Research 2011; 45: 1247-1257.

81. Corsi SR, et al. Human and bovine viruses in the Milwaukee River watershed: Hydrologically relevant representation and relations with environmental variables. Science of the Total Environment 2014; 490: 849-860.

82. Rose JB, Haas CN, Regli S. Risk assessment and control of waterborne giardiasis. American Journal of Public Health 1991; 81: 709-713.

83. Messner MJ, Chappell CL, Okhuysen PC. Risk assessment for Cryptosporidium: a hierarchical bayesian analysis of human dose response data. Water Research 2001; 35: 3934-3940.

84. Schmidt PJ, et al. Harnessing the theoretical foundations of the exponential and beta-poisson doseresponse models to quantify parameter uncertainty using Markov Chain Monte Carlo. Risk Analysis 2013; 33: 1677-1693.

85. Teunis PFM, Ogden ID, Strachan NJ. Hierarchical dose response of E. coli O157:H7 from human outbreaks incorporating heterogeneity in exposure. Epidemiology and Infection 2007; 136: 761-770.

86. Bielaszewska M, et al. Human Escherichia coli $\mathrm{O} 157: \mathrm{H} 7$ infection associated with the consumption of unpasteurized goat's milk. Epidemiology and Infection 1997; 119: 299-305.

87. Teunis PFM, et al. Norwalk virus: How infectious is it? Journal of Medical Virology 2008; 80: 1468-1476.

88. WHO (2004). Water Treatment and Pathogen Control. Geneva: World Health Organization, IWA Publishing. 